\title{
SIMULAÇÃO DE UM MODELO DE REATOR DE HIDRÓLISE ENZIMÁTICA DA BIOMASSA DE CANA-DE-AÇÚCAR OPERANDO EM BATELADA ALIMENTADA
}

\author{
E. H. SILVA ${ }^{1}$, A. R. A. LINO², F. FURLAN ${ }^{1}$ e R. C. GIORDANO ${ }^{2}$ \\ ${ }^{1}$ Universidade Federal de São Carlos, Departamento de Engenharia Química \\ ${ }^{2}$ Universidade Federal de São Carlos, Programa de Pós-Graduação em Engenharia \\ E-mail para contato: ewertonhs@ @otmail.com
}

\begin{abstract}
RESUMO - A utilização de modelagem e simulação de processos é importante para o desenvolvimento de novos processos e otimização de processos já existentes. Deste modo, o objetivo deste trabalho foi a simulação de um modelo de reator de hidrólise enzimática da biomassa de cana-de-açúcar operando em batelada alimentada, descrito por Montaño (2013). O modelo cinético utilizado foi o de Michaelis-Menten com inibição pelo produto. As políticas de alimentação de enzimas e substrato utilizadas apresentaram como principais objetivos a diminuição do efeito de inibição pelo produto e manutenção da concentração de glicose constante, respectivamente. Foram obtidos resultados satisfatórios para os objetivos operacionais, alcançando uma alta concentração de glicose. Contudo, houve uma alta utilização de enzimas, podendo tornar o processo proposto inviável devido ao gasto excessivo de enzimas.
\end{abstract}

\section{INTRODUÇÃO}

A intensificação do efeito estufa ocasionada pelo uso excessivo dos combustíveis fósseis, juntamente com a futura escassez das matérias-primas destes combustíveis, tem impulsionado o desenvolvimento de tecnologias sustentáveis. Nesse contexto, insere-se o bioetanol, um combustível largamente produzido e utilizado no Brasil (Sonego et al., 2016). No Brasil, o etanol é produzido a partir do caldo da cana-de-açúcar ou da mistura entre o caldo de cana e do melaço, empregando a levedura Saccharomyces cerevisiae como agente de fermentação. Um dos principais subprodutos desta produção é o bagaço (Amorin et al., 2011). O bagaço da cana-de-açúcar é constituído por aproximadamente $30-40 \%$ de celulose, $25-35 \%$ de hemicelulose, $20-30 \%$ de lignina, além de outros compostos. Após uma etapa de prétratamento, na qual a hemicelulose e/ou a lignina são separadas da celulose, esta pode ser hidrolisada gerando açúcares fermentescíveis (Dias, 2008). O etanol produzido a partir de materiais lignocelulósicos, como é o caso do bagaço, é comumente conhecido como etanol de segunda geração $(2 \mathrm{G})$.

Tal processo ainda se encontra em fase de desenvolvimento e possui diversos gargalos técnicos e econômicos. Um deles é a baixa concentração de açúcares resultantes da etapa de hidrólise, o que impacta no consumo de energia do restante do processo. Isso pode ser contornado com a operação do reator de hidrólise com altas cargas de sólidos. A hidrólise enzimática utilizando alta carga de sólidos é uma forma interessante de processamento, pois 
possibilita a redução dos custos de operação e da quantidade de água utilizada e o aumento da concentração de produto (Hodge et al., 2008). Porém, a operação em batelada de reatores com altas cargas de sólidos implica em altos consumos de energia com agitação e problemas difusionais. Assim, uma alternativa é sua operação em batelada alimentada, empregando políticas de alimentação racionais de substratos e catalisadores para o reator. Com isso, podese operar com cargas de sólidos mais baixas e ainda assim conseguir altas concentrações de produto (Montaño, 2013).

Neste contexto, este trabalho teve como objetivo a simulação, no simulador EMSO (Environment for Modeling Simulation and Optimization), de um reator de hidrólise operando em batelada alimentada utilizando o modelo cinético de Michaelis-Menten e empregando a política de alimentação de substrato e enzimas descrita por Montaño (2013). Com isso, foi possível avaliar o desempenho do reator em diferentes condições de operação.

\section{METODOLOGIA}

\subsection{Modelagem do reator de hidrólise enzimática}

Para o estudo em questão, foi utilizado o modelo cinético de Michaelis-Menten com inibição pelo produto (Equação 1), empregando os parâmetros cinéticos descritos por Montaño (2013). Neste caso, trata-se de um modelo não estruturado, isto é, não há adsorção da enzima na lignina e considera-se o conjunto de celulases como um único catalisador.

$$
\mathrm{r}=\frac{\mathrm{k} \cdot \mathrm{Ce} \cdot \mathrm{Cs}}{k_{m} \cdot\left(1+\frac{C p}{k_{i}}\right)+C s}
$$

$\mathrm{Na}$ Equação 1, Cs, Cp e Ce são as concentrações de substrato, produto e enzimas (g.L ${ }^{-}$ $\left.{ }^{1}\right)$, respectivamente, $\mathrm{r}$ é a taxa de reação $\left(\mathrm{g} \cdot \mathrm{L}^{-1} \cdot \mathrm{min}^{-1}\right), \mathrm{k}$ é a constante de velocidade de hidrólise da celulose $\left(\mathrm{min}^{-1}\right), \mathrm{k}_{\mathrm{m}}$ é a constante de Michaelis-Menten $\left(\mathrm{g} . \mathrm{L}^{-1}\right)$ e $\mathrm{k}_{\mathrm{i}}$ é a constante de inibição competitiva pela glicose $\left(\mathrm{g} \cdot \mathrm{L}^{-1}\right)$. Os valores dos parâmetros cinéticos utilizados foram $\mathrm{k}=0,0144 \mathrm{~min}^{-1}, \mathrm{k}_{\mathrm{m}}=15 \mathrm{~g} . \mathrm{L}^{-1} \mathrm{e} \mathrm{k} \mathrm{k}_{\mathrm{i}}=4,5 \mathrm{~g} \cdot \mathrm{L}^{-1}$ (Montaño, 2013).

Foi realizada a modelagem matemática para um processo em batelada alimentada. As Equações 2, 3 e 4 apresentam os balanços de massa para substrato, produto e a variação do volume, respectivamente.

$$
\begin{aligned}
& \frac{\mathrm{dCs}}{\mathrm{dt}}=\frac{F_{a \lim } \cdot C s_{a \lim }}{V}-r-\frac{\left(F_{a \lim }+F_{e}\right) \cdot C s}{V} \\
& \frac{\mathrm{dCp}}{\mathrm{dt}}=r-\frac{\left(F_{a \lim }+F_{e}\right) \cdot C p}{V} \\
& \frac{\mathrm{dV}}{\mathrm{dt}}=F_{a \lim }+F_{e}
\end{aligned}
$$


Sendo $F_{\text {alim }}\left(L \cdot \operatorname{Lin}^{-1}\right)$ a vazão volumétrica de alimentação de substrato, $F_{e}\left(L \cdot m^{-1}{ }^{-1}\right)$ a vazão volumétrica de enzimas, Csalim $\left(\mathrm{g} . \mathrm{L}^{-1}\right)$ a concentração de celulose da corrente de alimentação de substrato e V o volume de solução dentro do reator (L).

\subsection{Política de alimentação de substrato e enzima}

A política de alimentação do reator utilizada neste estudo teve como objetivo a produção de altas concentrações de glicose, com adição de enzima e substrato ao reator. $\mathrm{O}$ intuito de adicionar enzimas é compensar a inibição devido a presença de glicose. Essa política de alimentação foi proposta por Montaño (2013).

A Equação 5 representa a vazão de alimentação de substrato.

$$
F_{a \lim }=\frac{r \cdot V}{C s_{a \lim }-C s_{0}}
$$

As Equações 6 e 7 representam a alimentação de enzimas.

$$
\begin{gathered}
C e_{2}=\frac{r_{\text {inicial }} \cdot\left[k_{m} \cdot\left(1+\frac{C p}{k_{i}}\right)+C s_{0}\right]}{k \cdot C s_{0}} \\
e_{a \lim }=\left(C e_{2} \cdot V-C e \cdot V\right) \cdot \frac{0,85}{7,83 \cdot 108}
\end{gathered}
$$

Sendo $\mathrm{Ce}_{2}$ a concentração de enzimas necessária para manter a velocidade de reação no valor inicial $\left(\mathrm{g} \cdot \mathrm{L}^{-1} \cdot \mathrm{min}^{-1}\right)$ e ealim a atividade enzimática adicionada ao reator (L). Para conversão de unidade de massa para unidade de volume, sabe-se que 0,85 FPU/mL de enzima equivale a 7,83 g/L e há 108 FPU por mL de complexo enzimático (Montaño, 2013).

A alimentação de substrato na taxa descrita pela Equação 5 faz com que a concentração de substrato no período de alimentação (até que o volume máximo do reator seja atingido) permaneça constante. Já a alimentação de enzima, como citado anteriormente, tem como objetivo contornar a inibição pela glicose. Assim, a simulação foi realizada fazendo com que a concentração de enzimas no reator ficasse com o valor determinado pela Equação 6 quando a taxa de reação fosse menor do que $60 \%$ do valor inicial.

\subsection{Software EMSO}

A modelagem proposta foi simulada utilizando o software EMSO. Este é um simulador e otimizador de processos orientado a equações e pode operar tanto em regime estacionário como dinâmico.

Uma das características deste software é a acessibilidade aos modelos matemáticos desenvolvidos e a possibilidade de inserção de novos modelos ou aprimoramento daqueles já existentes. O EMSO utiliza uma linguagem de modelagem orientada a objetos (denominados devices), o que permite a organização estruturada das unidades que compõem o processo. A 
plataforma do EMSO possui uma interface gráfica na qual o usuário pode modelar e simular processos complexos pela simples seleção e conexão de modelos de cada equipamento (RODRIGUES et al., 2008).

\subsection{Simulações}

As simulações realizadas neste estudo foram comparadas com os resultados obtidos por Montaño (2013), que em seu estudo, a autora utilizou o software Matlab para a condução de suas simulações.

As condições empregadas para as simulações estão demonstradas na Tabela 1.

Tabela 1 - Condições empregadas para a simulação em batelada alimentada (MONTAÑO, 2013)

\begin{tabular}{|c|c|c|c|c|}
\hline $\begin{array}{c}\text { Volume inicial } \\
(\mathrm{L})\end{array}$ & $\begin{array}{c}\text { Volume Final } \\
(\mathrm{L})\end{array}$ & $\mathrm{Cs}_{0}\left(\mathrm{~g} \cdot \mathrm{L}^{-1}\right)$ & $\mathrm{Ce}_{0}\left(\mathrm{~g} \cdot \mathrm{L}^{-1}\right)$ & $\mathrm{Cs}_{\mathrm{alim}}\left(\mathrm{g} . \mathrm{L}^{-1}\right)$ \\
\hline \hline 0,05 & 0,6 & 44,44 & 7,83 & 173,04 \\
\hline
\end{tabular}

\section{RESULTADOS E DISCUSSÃO}

A partir da modelagem descrita na seção 2, foram obtidas as simulações dos perfis de velocidade de consumo de substrato, volume, concentração de substrato, produto, vazão de alimentação de enzimas e vazão de alimentação de substrato, como demonstra a Figura 1.

A política de alimentação proposta por Montaño (2013) visou manter a concentração de substrato constante durante todo o período de batelada alimentada. Porém, pode-se observar na Figura 1-A que houve um pequeno decréscimo na concentração deste durante a reação. Isso ocorreu pelo fato do efeito de diluição do substrato pela adição de enzima ter sido desconsiderado no cálculo da vazão de alimentação do substrato, pois sua consideração levou a um perfil com variações bruscas na alimentação, de difícil implementação em um processo real.

Por se tratar de um modelo com inibição pelo produto há um decréscimo da velocidade de reação com o tempo, como pode ser observado na Figura 1 - B, mesmo com a concentração de substrato praticamente constante. Como descrito na sessão 2.2 , quando a velocidade da reação atinge $60 \%$ de sua velocidade inicial, ocorre a adição de quantidade de enzimas (calculada pelas Equações 6 e 7) suficiente para que a velocidade retorne a seu valor inicial (Figura 1 - C). Esse comportamento é observado para as primeiras duas alimentações de enzimas (Figura 1 - B). Porém, observa-se na Figura 1 - B, que na terceira alimentação de enzimas a velocidade não consegue retornar ao seu valor inicial. Isso acontece, pois nesse estágio da batelada, a vazão de alimentação de substrato está bastante elevada (Figura 1-F) e, assim, a concentração de enzimas não consegue atingir o valor desejado. Com isso, foi observada uma alimentação contínua de enzimas nesta etapa, como mostra a Figura 1 - C.

O volume (Figura 1 - D) apresentou uma característica exponencial na etapa de alimentação do reator, o que era esperado, já que a vazão de alimentação de substrato (Equação 5) é proporcional ao próprio volume. 
Figura 1 - Simulação do reator de hidrólise em batelada alimentada. (A) Velocidade de reação, (B) Volume do reator, (C) Concentração de substrato, (D) Concentração de produto, (E) Vazão de alimentação de enzima e (F) Vazão de alimentação de substrato.

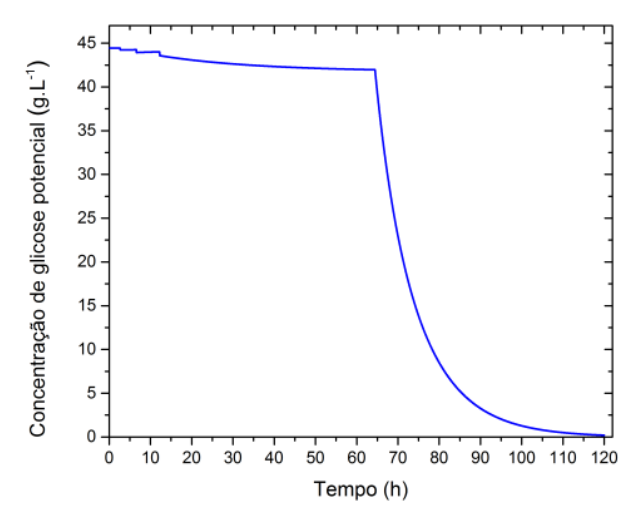

(A)

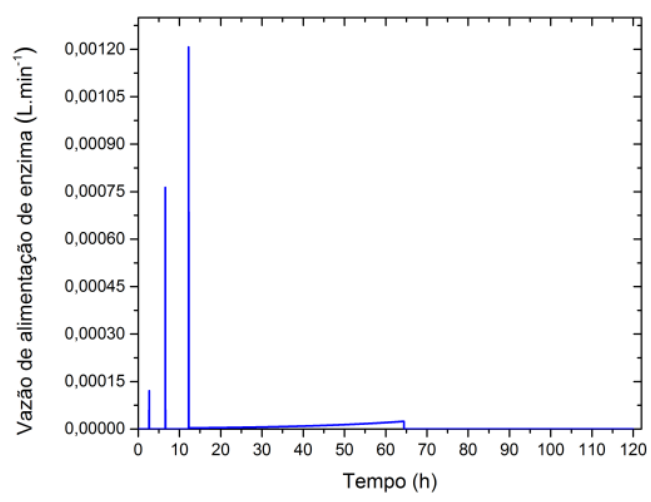

(C)

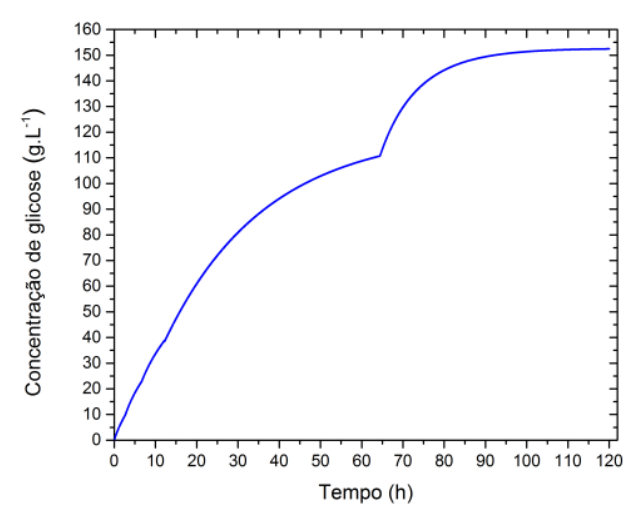

(E)

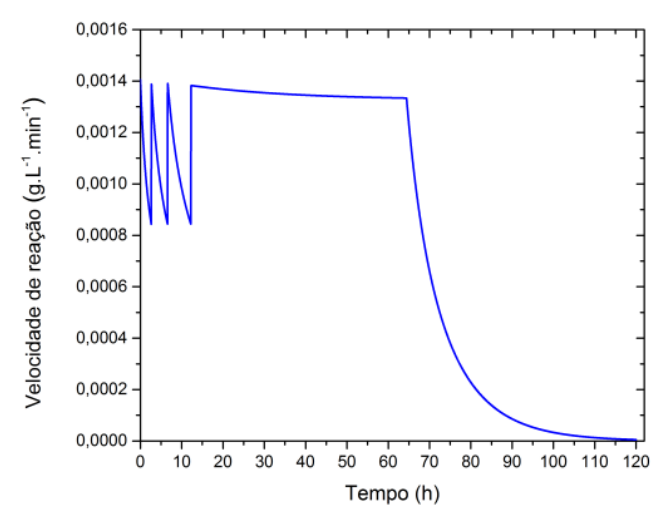

(B)

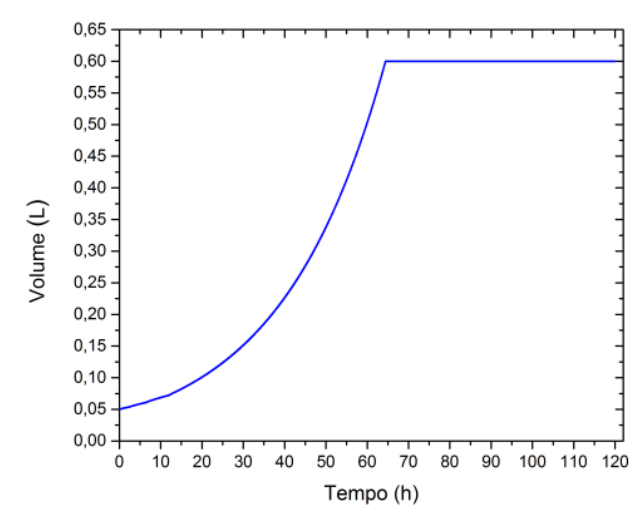

(D)

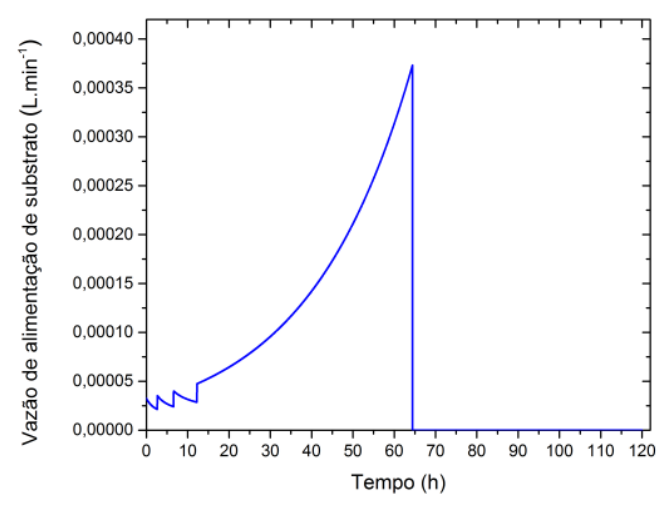

(F)

A concentração de glicose no reator, observada na Figura 1 - E, apresenta aumento em ambas as etapas do processo, demonstrando assim, que a taxa de produção de substrato supera a diluição ocasionada pela alimentação de enzimas e de substrato. Contudo, observa-se que há 
um acréscimo maior na etapa de batelada alimentada, o que é esperado, pois nesse período a velocidade da reação é maior, na média, do que na etapa de batelada.

É importante ressaltar que os resultados obtidos no presente estudo não foram de total concordância com os resultados obtidos por Montaño (2013). Os picos de alimentação de enzimas apresentaram valores diferentes e também foram realizados em tempos diferentes. Deste modo, toda a dinâmica foi afetada. Porém, o valor final de concentração de produto ficou bastante próximo deste.

\section{CONCLUSÃO}

A partir dos resultados obtidos pode-se concluir que o trabalho proposto conseguiu reproduzir de forma satisfatória o comportamento da batelada alimentada descrita por Montaño (2013), chegando a valores próximos de concentração de glicose ao final da batelada. Porém, vale ressaltar que a politica de alimentação descrita neste trabalho não consiste em uma solução ótima, visto que devido ao aumento exponencial do volume, a alimentação de enzimas torna-se contínua após um certo período, o que ocasionaria um gasto excessivo com enzimas e possivelmente levaria a resultados econômicos desfavoráveis.

\section{REFERÊNCIAS BIBLIOGRÁFICAS}

AMORIM, H. V.; LOPES, M. L.; OLIVEIRA, J. V. C.; BUCKERIDGE, M. S.; GOLDMAN, G. H. Scientific challenges of bioethanol production in Brazil. Applied Microbiology Biotechnology, v. 91, n. 5, p. 1267, Jul. 2011.

DIAS, M. O. S. Simulação do processo de produção de etanol a partir do açúcar e do bagaço, visando a integração do processo e a maximização da produção de energia e excedentes do bagaço. UNICAMP, Campinas, SP, 2008 (Dissertação). 253p.

HODGE, D. B.; KARIM, M. N.; SCHELL, D. J.; MCMILLAN, J. D. Soluble and insoluble solids contributions to high-solids enzymatic hydrolysis of lignocellulose. Bioresource Technology, v. 99, n. 18, p. 8940-8948, 2008.

MONTAÑO, I. D. C. Aplicação de técnicas de modelagem e simulação para a produção de etanol de segunda geração. 2013. 125 f. Tese (Doutorado) - Curso de Engenharia Química, Universidade Federal de São Carlos, São Carlos, 2013.

RODRIGUES, R.; SOARES, R. P.; SECCHI, A. R. Coleção de 10 problemas numéricos típicos em engenharia química resolvidos com o simulador EMSO. In: Anais do XVII Congresso Brasileiro de Engenharia Química (COBEQ), Recife, 2008.

SONEGO, J. L. S.; LEMOS, D. A.; PINTO, C. E. M.; CRUZ, A. J. G.; BADINO, A. C. Extractive Fed-Batch Ethanol Fermentation with CO2Stripping in a Bubble Column Bioreactor: Experiment and Modeling. Energy \& Fuels, São Carlos, v. 30, n. 1, p.748757, 21 jan. 2016. 